Journal of Patient-Centered

\title{
Relationship of Patient Self-Administered COPD Assessment Test to Physician Standard Assessment of Chronic Obstructive Pulmonary Disease in a Family Medicine Residency Training Program
}

Jessica E. Burchette

Ivy A. Click

Leigh Johnson

S. Alicia Williams

Brett Tyler Morgan

Follow this and additional works at: https://aah.org/jpcrr

Part of the Analytical, Diagnostic and Therapeutic Techniques and Equipment Commons, Cardiovascular Diseases Commons, Family Medicine Commons, Medical Education Commons, Pulmonology Commons, and the Respiratory Tract Diseases Commons

\section{Recommended Citation}

Burchette JE, Click IA, Johnson L, Williams SA, Morgan BT. Relationship of patient self-administered COPD Assessment Test to physician standard assessment of chronic obstructive pulmonary disease in a family medicine residency training program. J Patient Cent Res Rev. 2019;6:210-5. doi: 10.17294/ 2330-0698.1699

Published quarterly by Midwest-based health system Advocate Aurora Health and indexed in PubMed Central, the Journal of Patient-Centered Research and Reviews (JPCRR) is an open access, peer-reviewed medical journal focused on disseminating scholarly works devoted to improving patient-centered care practices, health outcomes, and the patient experience. 


\title{
Relationship of Patient Self-Administered COPD Assessment Test to Physician Standard Assessment of Chronic Obstructive Pulmonary Disease in a Family Medicine Residency Training Program
}

\author{
Jessica E. Burchette, PharmD, ${ }^{1}$ Ivy A. Click, EdD, ${ }^{2}$ Leigh Johnson, MD, ${ }^{2}$ S. Alicia Williams, MA, ${ }^{2}$ \\ Brett Tyler Morgan, MA $^{2}$ \\ ${ }^{1}$ Department of Pharmacy Practice, Bill Gatton College of Pharmacy, East Tennessee State University, Johnson City, TN; \\ ${ }^{2}$ Department of Family Medicine, Quillen College of Medicine, East Tennessee State University, Johnson City, TN
}

\begin{abstract}
Assessing the global impact of chronic obstructive pulmonary disease (COPD) on a patient's life can be difficult to perform in the clinical setting due to time constraints and workflow challenges. The primary objective of this study was to compare disease impact ratings between patient selfadministered COPD Assessment Test (CAT) and physician standard office assessment. This prospective study was conducted at a family medicine residency clinic in northeast Tennessee. The study included two study groups: 1) adult patients seen at the clinic during the 3-month study period with an active diagnosis of COPD, and 2) their physicians. Physicians' assessment of the impact of COPD on their patients' daily lives was compared to patients' self-administered CAT assessments. Physician assessment of COPD impact and patient assessment of CAT categories significantly differed $\left(X^{2}=11.0, P=0.012\right)$. There was very poor agreement between patient and physician ratings ( $\mathrm{k}=0.003$ ), with $42.9 \%$ of physician ratings underestimating the impact, $28.6 \%$ overestimating the impact, and $28.6 \%$ correctly estimating the impact COPD had on their patients' lives. These findings support the use of validated assessment tools to help providers understand the symptom burden for patients with COPD. (J Patient Cent Res Rev. 2019;6:210-215.)
\end{abstract}

Keywords chronic obstructive pulmonary disease; COPD; assessment test; quality of life; family medicine

A $\mathrm{n}$ estimated 11 million Americans have a diagnosis of chronic obstructive pulmonary disease (COPD), with another 13 million potentially affected. ${ }^{1,2}$ Currently, COPD is ranked as the third-leading cause of death in the United States. ${ }^{3}$ Certain geographical regions are more impacted by COPD due in part to cultural influences and lifestyle factors such as smoking and occupational exposure. Tennessee has one of the higher percentages of citizens

Correspondence: Jessica E. Burchette, PharmD, East Tennessee State University, PO Box 70657, Johnson City, TN 37614 (burchettej@etsu.edu) diagnosed with COPD, with a current prevalence estimate of $8.1 \%$ (state range: $3.5 \%-13.6 \%$ ). ${ }^{1}$

Symptoms of COPD are highly variable with significantly different presentations between patients. ${ }^{4,5}$ Hallmark symptoms of shortness of breath and increased sputum production can often overshadow nonspecific symptoms, which may be overlooked or not attributed to COPD. Anxiety, depression, and difficulty sleeping are often present but may not be correlated to COPD by patients or providers. ${ }^{6}$ These symptoms can be difficult to measure and often affect activities of daily living as well as confidence in simple tasks such as leaving the home without fear of worsened symptoms. ${ }^{7}$ 
Assessing the global impact of COPD on an individual patient can be difficult to perform because of time constraints and workflow challenges in clinic. ${ }^{8}$ Also, patients may underreport the severity of COPD during standard patient-provider interactions due to the variability of symptoms over time. ${ }^{9}$ Given that ample data have indicated that spirometry does not directly correlate with symptoms, ${ }^{10}$ the need for a reliable assessment tool to measure disease impact is crucial.

Family medicine providers care for a complex and diverse patient population with many chronic diseases. Utilizing a standard assessment to guide the patient discussion can assist providers in formulating evidence-based care plans for individual patients. These assessments can open discussion regarding disease impact on quality of life beyond a generic "How are you?" approach. ${ }^{11}$ Understanding and valuing which aspects of a disease are most concerning to patients is a cornerstone of patientcentered care and has an influence on patient-provider trust and rapport. ${ }^{12}$

The COPD Assessment Test (CAT) was developed in 2009 as a short, patient-administered questionnaire for measuring health-related quality of life for patients with COPD. ${ }^{13}$ This assessment was designed for clinical practice, taking about 2 to 3 minutes to complete, and has been validated in multiple languages. ${ }^{14}$ Ideally, patients would self-administer the CAT prior to an office visit as a tool for stimulating discussion. Scores range from 0 to 40 and are categorized as low, medium, high, or very high impact. The CAT is currently undergoing review by an expert governance board to ensure usability and reliability for patients everywhere. ${ }^{15}$

The primary objective of this study was to compare disease impact ratings between patient self-administered CAT and physician standard office assessment. Secondary objectives include whether patient and/or physician characteristics impacted COPD assessment scoring variations between the two groups.

\section{METHODS}

This prospective study was conducted at a family medicine residency clinic in northeast Tennessee. The study included two groups: 1) adult patients seen at the clinic during the 3-month study period with an active ICD-10 code consistent with COPD, and 2) their physicians. This study was approved by the East Tennessee State University institutional review board. Informed consent was obtained from all participants. The clinic's social health specialist reviewed a weekly report of scheduled appointments and assessed for the following inclusion criteria: age of $\geq 18$ years, active diagnosis of COPD, English speaking, legally able to make personal treatment decisions, and not pregnant at the time of the visit. Those with an acute exacerbation of COPD within 2 weeks of the visit were excluded. Potential patients were highlighted each day prior to the start of clinic. All clinic physicians were eligible for inclusion.

Upon completion of the visit, physicians completed a 1-item assessment of their perception of whether COPD had a low, medium, high, or very high impact on their patient's quality of life (mimicking CAT impact categories). Physician gender and experience level (resident training year, faculty) were collected. Eligible patients were approached by the nurse before leaving the examination room to ask about willingness to participate. Those willing to participate were escorted to the social health specialist's office, where they were consented and asked to complete the CAT. Patient demographics, including age, gender, and smoking status, were collected. Figure 1 illustrates the study workflow.

Descriptive analyses were used to provide summaries of patient and physician participant demographic characteristics. Patient participants' CAT scores were totaled and categorized by impact on wellbeing and daily life (low, medium, high, or very high impact). Chi-squared $\left(\chi^{2}\right)$ tests were used to compare physicians' ratings of COPD impact to their patients' CAT categories, as well as differences in sex, smoking status, and physician experience. Cohen's kappa (к) coefficient was used to compare agreement between physician and patient ratings. All analyses were conducted using SPSS Statistics, Version 23 (IBM Corp, Armonk, NY).

\section{RESULTS}

Fifty matched pairs of patient CAT and physician assessment forms were collected over 3 months. One set was removed due to incomplete data, leaving 49 


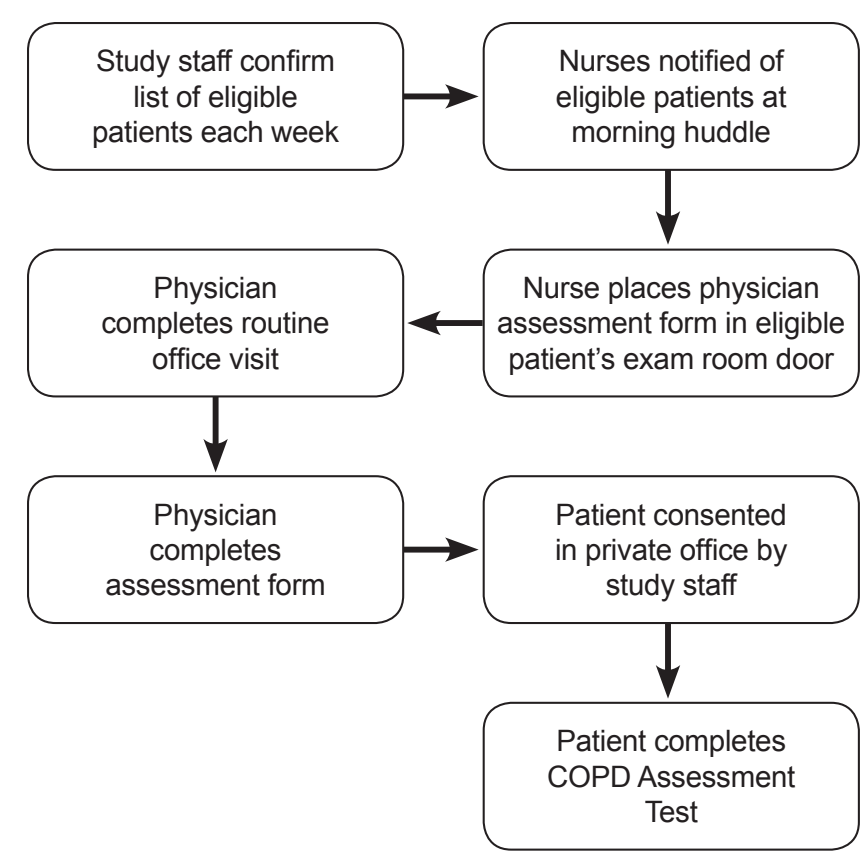

Figure 1. Chronic obstructive pulmonary disease (COPD) assessment study flow.

usable sets of matched forms. The majority of the 49 patient participants were female $(61.2 \%)$, and the cohort averaged $63.5 \pm 8.9$ years of age. Slightly more than half were current smokers (51\%). Physician participants were more frequently male $(55.1 \%)$ and third-year family medicine residents (40.8\%) (Table 1).

There were significant differences between physician assessment of COPD impact on patients' lives and patients' self-assessment of CAT categories $\left(\chi^{2}=11.0, \mathrm{P}=0.012\right)$ (Figure 2). Data indicated very poor agreement between patient and physician ratings $(\kappa=0.003)$, with $42.9 \%$ of physician ratings underestimating the impact, $28.6 \%$ overestimating the impact, and $28.6 \%$ correctly estimating the impact COPD had on their patients' lives. Furthermore, physicians were more likely to underestimate COPD impact for those not currently smoking (50\%) versus current smokers $(36.0 \%) ; \mathrm{P}=0.047$. Although female physicians were more likely to correctly assess COPD impact (40.9\%) than male physicians (18.5\%), this was not statistically significant $(\mathrm{P}=0.08)$. There were no significant differences based on physician experience, patient sex, or patient age.

\section{DISCUSSION}

Implementing a standardized, validated assessment tool to monitor chronic disease impact has value for patients and providers. ${ }^{9}$ Our study identified a discrepancy between physician assessment of disease impact and patient self-reported impact via CAT scoring. One explanation for this incongruity is a lack of general knowledge among physicians and patients of less welldescribed COPD symptoms. Most patients understand that shortness of breath, coughing, and sputum production are symptoms of COPD; however, other symptoms such as anxiety, depression, or poor sleep patterns may not be considered COPD-related. Patients with COPD have been shown to experience a higher mental health burden than patients with other chronic health conditions, and the correlation between COPD and mental health has been linked to negative effects on mortality, increased risk of exacerbations, longer hospital stays, and poorer quality of life with decreased functional status. ${ }^{16,17}$ During a general patient encounter, the patient may not report psychological symptoms when asked about the impact of COPD on daily life because of a lack of knowledge related to symptom-disease relationship. ${ }^{18}$ Clinically, there are significant implications for patients to underestimating the impact of COPD, including potential undertreatment of symptoms that could lead to acute exacerbations, further decreasing quality of life.

Another potentiating factor in the patient-provider disease assessment may be variation between individual providers related to COPD assessment. For example, female physicians are more likely to engage in patientcentered communication, ${ }^{19,20}$ suggesting that perhaps they are better poised to estimate individual patient disease impact. The increased correct assessment of COPD impact by female physicians was not statistically significant in this pilot study, perhaps due to sample size. Nonetheless, as each provider has his or her own style of patient interview, the assessment of disease may vary based on the types of questions asked and the way in which they are posed to the patient. By implementing a standardized assessment for COPD impact, patients and providers can come to the encounter with common ground for assessment and discussion.

The clinical impact of adding a standardized assessment of COPD, such as the CAT, to routine care for all patients with COPD does not come without added 
Table 1. Characteristics of Patients $(n=49)$ and Physicians $(n=49)$ and Their Respective Relationships to COPD Impact Agreement

\begin{tabular}{|c|c|c|c|c|c|}
\hline \multirow[b]{2}{*}{ Characteristic } & \multirow[b]{2}{*}{$\begin{array}{c}\text { All, } \\
\text { n (\%) }\end{array}$} & \multicolumn{3}{|c|}{ Physician Agreement With Patient CAT } & \multirow[b]{2}{*}{$\boldsymbol{P}$} \\
\hline & & $\begin{array}{c}\text { Same, } \\
\mathrm{n}(\%)\end{array}$ & $\begin{array}{c}\text { Under, } \\
\text { n (\%) }\end{array}$ & $\begin{array}{l}\text { Over, } \\
\text { n (\%) }\end{array}$ & \\
\hline COPD impact & & $14(28.6)$ & $21(42.9)$ & $14(28.6)$ & 0.01 \\
\hline \multicolumn{6}{|l|}{ Patient age* } \\
\hline $45-54$ years & $10(20.4)$ & $5(50.0)$ & $4(40.0)$ & $1(10.0)$ & \multirow{4}{*}{0.36} \\
\hline $55-64$ years & $14(28.6)$ & $3(21.4)$ & $5(35.7)$ & $6(42.9)$ & \\
\hline $65-74$ years & $20(40.8)$ & $4(20.0)$ & $11(55.0)$ & $5(25.0)$ & \\
\hline $75-84$ years & $5(10.2)$ & $2(40.0)$ & $1(20.0)$ & $2(40.0)$ & \\
\hline \multicolumn{6}{|l|}{ Patient sex } \\
\hline Female & $30(61.2)$ & $7(23.3)$ & $14(46.7)$ & $9(30.0)$ & \multirow{2}{*}{0.31} \\
\hline Male & $19(38.8)$ & $7(36.8)$ & $7(36.8)$ & $5(26.3)$ & \\
\hline \multicolumn{6}{|l|}{ Physician sex } \\
\hline Female & $22(44.9)$ & $9(40.9)$ & $8(36.4)$ & $5(22.7)$ & \multirow{2}{*}{0.08} \\
\hline Male & $27(55.1)$ & $5(18.5)$ & $13(48.1)$ & $9(33.3)$ & \\
\hline \multicolumn{6}{|c|}{ Patient smoking status } \\
\hline Current smoker & $25(51.0)$ & $11(44.0)$ & $9(36.0)$ & $5(20.0)$ & \multirow[b]{2}{*}{0.05} \\
\hline Former smoker & $24(49.0)$ & $3(12.5)$ & $12(50.0)$ & $9(37.5)$ & \\
\hline \multicolumn{6}{|c|}{ Physician training year } \\
\hline PGY 1 & 8 (16.3) & $2(25.0)$ & $4(50.0)$ & $2(25.0)$ & \multirow{4}{*}{0.47} \\
\hline PGY 2 & $18(36.7)$ & $8(44.4)$ & $6(33.3)$ & $4(22.2)$ & \\
\hline PGY 3 & $20(40.8)$ & $4(20.0)$ & $10(50.0)$ & $6(30.0)$ & \\
\hline Faculty & $3(6.1)$ & $0(0.0)$ & $1(33.3)$ & $2(66.7)$ & \\
\hline
\end{tabular}

*Mean age for the 49-patient cohort was $63.5 \pm 8.9$ years.

CAT, COPD Assessment Test; COPD, chronic obstructive pulmonary disease; PGY, postgraduate year.

time requirements for clinical workflow. Practice administrators should evaluate current procedures to determine appropriate places within the patient/clinic flow to administer these assessments. Patient checkin is an easily identifiable point in clinic workflow to administer an assessment; this procedure would allow patients to complete the assessment prior to seeing the provider and may prompt patients to generate questions regarding their care. To avoid the potential of biasing the study, our patients did not complete the CAT prior to seeing the provider, but in daily practice asking a patient to complete the CAT prior to the visit would likely be more beneficial to the patientprovider discussion. ${ }^{14}$ Alternatively, asking the nurse to provide the CAT to the patient as they are roomed could provide more information during the encounter without disrupting clinic flow.
This study has many strengths, which support the validity of the results in practice. The study was a prospective review of real-time provider assessment of COPD impact on a patient's life. The providers were instructed to perform their assessment and exam as usual and had not been previously educated on the CAT. Patient self-assessment of disease impact via the CAT is validated in the literature and proven to increase the comprehensiveness of patient symptom reporting. ${ }^{9,21}$ Having patients complete the CAT after the encounter prevented discussion of the questions and results with the provider during the visit, helping to reduce study bias.

Although efforts were taken to solidify and strengthen this study, there are limitations that should be addressed. Due to patient identification methods and variation in monthly resident schedules, we were 


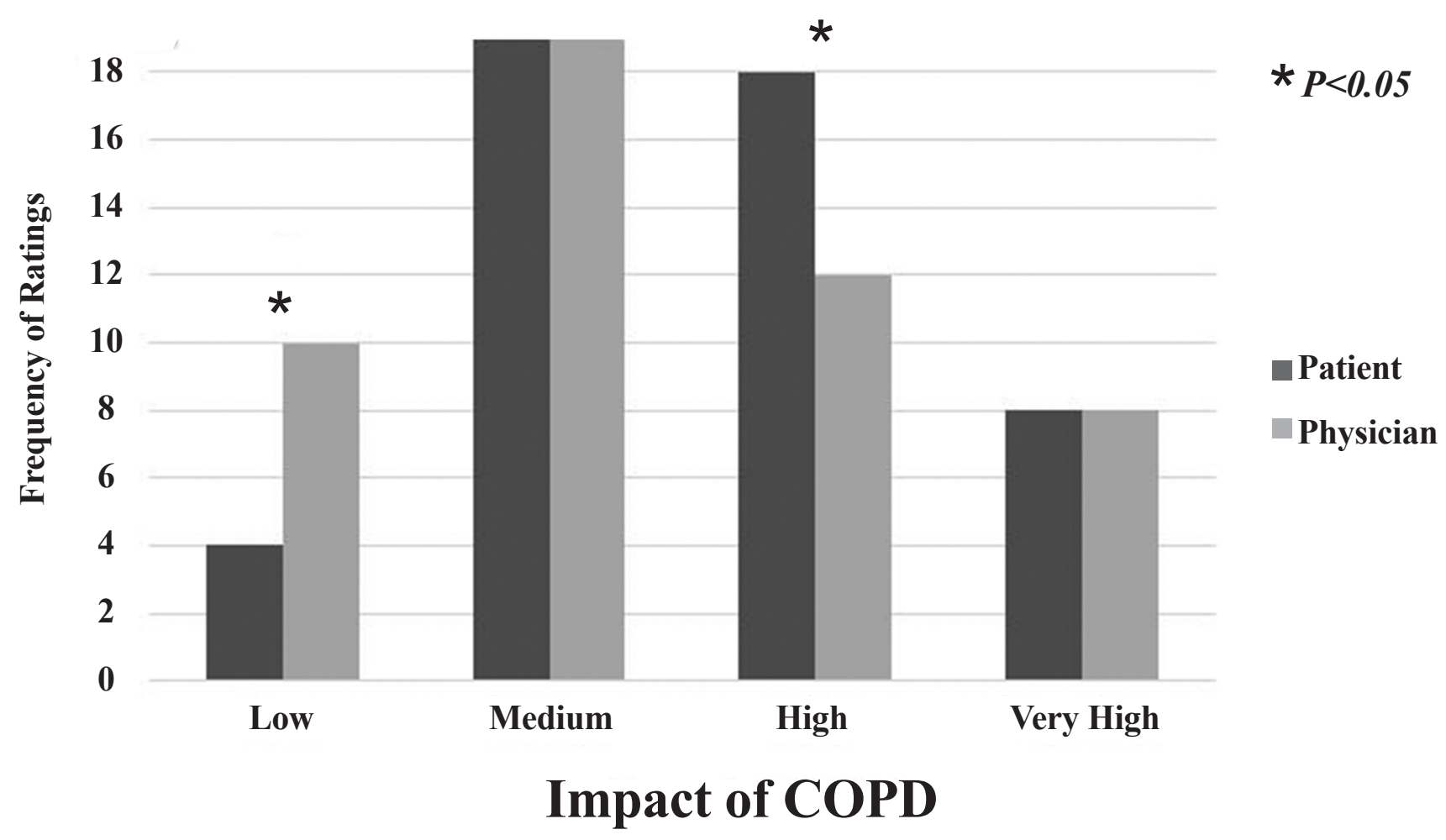

Figure 2. Comparison of patient COPD Assessment Test categories and physician assessment of chronic obstructive pulmonary disease (COPD) on well-being and daily life.

unable to assess the level of provider/patient continuity of care. Arguably, a lack of familiarity with the patient may have impacted the provider's ability to assess disease impact. Another limitation is the difference in assessment methods for comparing patient and provider results. Physicians were asked to categorize patients directly into 1 of 4 impact categories, whereas patient CAT scores were represented numerically as the sum of the 8-item Likert scale assessment and then categorized by impact. This difference in scoring could have created discrepancy between the provider and patient scoring. Finally, although we made multiple efforts to reduce study bias, it is possible physicians may have been alerted to a potential study participant prior to seeing the patient, which could have triggered the physician to ask additional COPD-related questions. However, even with this potential bias, we still found physicians underestimated the impact of COPD on patients' lives.

Future plans include assessing the effect of fully implementing the CAT into practice and the impact on treatment decisions. As addressed previously, there are no available data addressing variation among provider styles. Future work that attempts to quantify and qualify provider style differences may further contribute to the development of reliable standardized patient assessments. Much of the future direction regarding the widespread use of the CAT within the United States will depend on the outcomes from the CAT governance board. ${ }^{15}$

Our results indicated poor agreement between physicians' standard assessment of COPD symptom impact on patients' daily lives and patients' selfassessment. These findings support the use of validated assessment tools to help providers understand the symptom burden for patients with COPD. Family medicine providers are uniquely positioned to care for patients with many chronic diseases, which includes understanding the patients' perspective on disease and the symptom burden impacting daily life. Standardized measures of disease impact, such as the CAT, have value for both patients and providers in daily practice and can be an important tool in creating a dialogue 
between provider and patient. This study serves as an important pilot identifying a potential area for improvement in patient-provider communication in any clinic caring for patients with COPD.

\section{Patient-Friendly Recap}

- Chronic obstructive pulmonary disease, or COPD, can be a deadly condition but also results in a range of serious symptoms that negatively affect patients' lives.

- The authors compared the responses of patients to questions on a COPD selfassessment test with those of the patients' physicians in a clinical setting.

- They found that physicians often underestimate the presence of COPD symptoms and the disease's impact on patients' quality of life.

- This discrepancy may be, in part, due to patients not realizing certain symptoms, like anxiety or poor sleep, can be related to COPD and should be reported as such.

\section{Author Contributions}

Study design: Burchette, Click, Johnson, Williams. Data acquisition or analysis: Click, Williams, Morgan. Manuscript drafting: Burchette, Click, Johnson. Critical revision: Williams, Morgan.

\section{Conflicts of Interest}

None.

\section{References}

1. American Lung Association. How serious is COPD: COPD prevalence, 2017. Last updated 2019 Apr 5. http://www.lung. $\mathrm{org} /$ lung-health-and-diseases/lung-disease-lookup/copd/learnabout-copd/how-serious-is-copd.html. Accessed April 8, 2019.

2. Mannino DM, Homa DM, Akinbami LJ, Ford ES, Redd SC. Chronic obstructive pulmonary disease surveillance--United States, 1971-2000. MMWR Surveill Summ. 2002;51(6):1-16.

3. Kochanek KD, Murphy SL, Xu J, Tejada-Vera B. Deaths: final data for 2014. Natl Vital Stat Rep. 2016;65(4):1-122.

4. Kessler R, Partridge MR, Miravitlles M, et al. Symptom variability in patients with severe COPD: a pan-European cross-sectional study. Eur Respir J. 2011;37:264-72. CrossRef

5. Gilbert C, Martin ML, Hareendran A. Capturing individual variation in the experience of symptoms reported by patients with COPD. (abstr.) Am J Respir Crit Care Med. 2007; 175:A15-A1004.

6. Miravitlles M, Ribera A. Understanding the impact of symptoms on the burden of COPD. Respir Res. 2017;18(1):67. CrossRef
7. Gardiner C, Gott M, Payne S, et al. Exploring the care needs of patients with advanced COPD: an overview of the literature. Respir Med. 2010;104:159-65. CrossRef

8. Perez X, Wisnivesky JP, Lurslurchachai L, Kleinman LC, Kronish IM. Barriers to adherence to COPD guidelines among primary care providers. Respir Med. 2012;106:374-81. CrossRef

9. van der Molen T, Miravitlles M, Kocks JW. COPD management: role of symptom assessment in routine clinical practice. Int $J$ Chron Obstruct Pulmon Dis. 2013;8:461-71. CrossRef

10. Global Initiative for Chronic Obstructive Lung Disease (GOLD). Global Strategy for the Diagnosis, Management, and Prevention of Chronic Obstructive Pulmonary Disease (2018 Report). Fontana, WI: Global Initiative for Chronic Obstructive Lung Disease Inc, 2018, pp. 1-123.

11. Detmar SB, Muller MJ, Schornagel JH, Wever LD, Aaronson NK. Health-related quality-of-life assessments and patientphysician communication: a randomized controlled trial. JAMA. 2002;288:3027-34. CrossRef

12. Pumar MI, Gray CR, Walsh JR, Yang IA, Rolls TA, Ward DL. Anxiety and depression - important psychological comorbidities of COPD. J Thorac Dis. 2014;6:1615-31. CrossRef

13. Jones PW, Harding G, Berry P, Wiklund I, Chen WH, Kline Leidy N. Development and first validation of the COPD assessment test. Eur Respir J. 2009;34:648-54. CrossRef

14. COPD Assessment Test: healthcare professional user guide. Issue 4 updated 2018 Nov. https://www.catestonline.org/ content/dam/global/catestonline/documents/CAT_HCP\%20 User\%20Guide.pdf. Accessed April 8, 2019.

15. GlaxoSmithKline plc. GSK, GOLD and the COPD Foundation announce formation of a new external expert Governance Board for the COPD Assessment Test (CAT). Press release dated 2014 Sep 8. https://www.gsk.com/en-gb/media/press-releases/gskgold-and-the-copd-foundation-announce-formation-of-a-newexternal-expert-governance-board-for-the-copd-assessmenttest-cat/. Accessed September 26, 2017.

16. Dury R. COPD and emotional distress: not always noticed and therefore untreated. Br J Community Nurs. 2016;21:138-41. CrossRef

17. Celli B, Blasi F, Gaga M, et al. Perception of symptoms and quality of life - comparison of patients' and physicians' views in the COPD MIRROR study. Int J Chron Obstruct Pulmon Dis. 2017;12:2189-96. CrossRef

18. Miravitlles M, Ferrer J, Baro E, Lleonart M, Galera J. Differences between physician and patient in the perception of symptoms and their severity in COPD. Respir Med. 2013;107:1977-85. $\underline{\text { CrossRef }}$

19. Bertakis KD, Azari R. Patient-centered care: the influence of patient and resident physician gender and gender concordance in primary care. $J$ Womens Health (Larchmt). 2012;21:326-33. CrossRef

20. Roter DL, Hall JA, Aoki Y. Physician gender effects in medical communication: a meta-analytic review. JAMA. 2002;288:756-64. CrossRef

21. Gupta N, Pinto LM, Morogan A, Bourbeau J. The COPD assessment test: a systematic review. Eur Respir J. 2014;44:873-84. CrossRef

(C) 2019 Aurora Health Care, Inc. 